\author{
balance of the journal."
}

\title{
'Some exciting and significant innovations'
}

From the next issue of the British Dental Journal, on 13 January 2007, readers will notice a difference to the content and balance as we implement some exciting and significant innovations. These may be broadly summarised as a change to Advanced Online Publication (AOP) and to Electronic Long Paper Short (ELPS) delivery for the 'research' section of the journal.

\section{ADVANCED ON-LINE PUBLICATION (AOP)}

Currently, once a research paper has been accepted for publication in the $B D J$ there is a delay of between nine months and one year before it is published, which we believe to be unacceptable. Therefore, from January onwards, once a research paper has been accepted for publication, typeset, proofread by the author(s) and returned to us it will be published online straight away (www.bdj.co.uk). While there will inevitably be some minor variation in this process, we anticipate that accepted papers will be published online in less than two months from acceptance and we will continue to work on ways to reduce this still further.

\section{ELECTRONIC LONG PAPER SHORT (ELPS)}

Simultaneously we will be changing to the ELPS system. This means that research papers will be published in full online only and will no longer be published in full in the print version of the $B D J$, although we will reserve the capacity to do so if a paper is considered of particular merit or importance. In the place of the full research paper, each issue will contain enhanced summaries of two or three research papers (the full text of which will be online). The enhanced summaries will each be a double page spread and will include:

- The 'In brief' (as now)

- A commentary (as now)

- An editor's summary (new)

- A sentence or two from the authors explaining why they undertook the study/work (new)

- A sentence or two from the authors explaining what they would like to do as a follow on from their study/work (new)

- An indication of whether the full paper online is a CPD paper or not (new).

The Contents section of the print version of the $B D J$ will list all papers that have been put online since the previous issue so as to inform readers of, and alert them to, newly available material. The online table of contents will remain similar in appearance to the current version, with a research section where the papers are listed.

These changes will not affect the way in which papers are referenced or cited. $B D J$ papers will continue to be citable and will be included in Medline, PubMed, ISI and the other existing listing, database and referencing services. It may well be that the journal's Immediacy Factor will rise as a result of these changes, although the journal has rated highly on this measure for some years. It may also be that its Impact Factor will rise as a result of greater and faster online access.

The CPD programme will continue but in future one of the two 'CPD' papers in each issue will be a research paper, and therefore in full online only. This means that in order to answer the questions, participants will need to go online. We hope that as a result of this more readers will go online and explore the advantages of the $B D J$ site and of electronic publishing in general. As one of the CPD papers will still be in the print form, participants who for whatever reason do not want to go online to study the other paper, will still have the opportunity to accumulate 24 verifiable hours per year, which is in excess of the current requirement of an average of 15 hours.

We are making these changes following reader research, author feedback, detailed analysis of online data and awareness of developments in publishing both in print and electronically. Amongst the aims are:

- Faster publication and better access for authors and researchers

- Greater use of the $B D J$ in paper and electronic form by all readers

- To give the $B D J$ a new feel, tailored to the varying needs of the readership

- To provide more practical articles for practitioners, who form the majority of the readership.

We will of course be carefully monitoring the effect of these innovations in the coming months and welcome feedback on them. The aim is always to improve the journal and enhance its value and reputation.

Stephen Hancocks OBE, Editor-in-Chief doi: 10.1038/sj.bdj.4814366 